\title{
On High-precision Subpixel-based Drilling Technique for Suture Needles with Thread
}

\author{
Tiebo Sun ${ }^{*}$ and Hong Li \\ Mechanical and Electrical Engineering Department, Jiangsu Food \& Pharmaceutical Science College, Jiangsu \\ Huai'an, 223003, China
}

\begin{abstract}
In order to improve the automation of end-hole drilling process in the production of suture needles with thread, a high-precision subpixel-based drilling method is proposed. According to the edge detection principle in mathematical morphology, combined with the characteristics of the magnified images of the ends of suture needles to be drilled, the morphological edge detection operators with variable structural elements are constructed to achieve noise suppression and fully extract the detailed information of edges of images of needle end holes to be drilled. Then, the subdivision method of spatial moments is adopted to realize the subpixel positioning of pixel-level edges. Finally, least squares fitting method is used to achieve the high-precision positioning of center of needle end hole to be drilled. The experimental results of the $0.5 \mathrm{~mm}$ needle samples show that the drilling method proposed in this study has a concentricity error no more than \pm 0.2 $\mu \mathrm{m}$ and an average drilling time of $0.65 \mathrm{~S}$. Moreover, the method also boasts good real-time performance and stability and meets the automated production needs of drilling process of suture needles with thread.
\end{abstract}

Keywords: Least squares fitting, machine vision, mathematical morphology, subpixel, suture needles with thread.

\section{INTRODUCTION}

Medical suture needles are mainly used in the surgical suture of skin, subcutaneous tissues, fascia and muscle. With constant improvement of medical treatment level, higher requirements have been put forward for medical devices. In this context, medical suture needles have experienced three generations of development from the traditional eye needles, while slotted needles have been developed to suture needles with thread. Currently, suture needles with thread are the most widely surgical needles, which are manufactured by inserting the suture thread through the needle end and axle hole and getting it stuck. Suture needles with thread can greatly decrease the drag force of tissues in the suture process and reduce suture wounds. The suture needle diameter is $\phi 0.5 \sim 1.3 \mathrm{~mm}$ and the end-hole diameter is $\phi$ $0.25 \sim 0.65 \mathrm{~mm}$. A critical process in the production of suture needles with thread is end-hole drilling [1]. At present, the domestic manufacturers generally adopt manual drilling, while the expensive labor costs and a higher rejection rate resulting from small sizes largely affect the production efficiency of suture needles. Consequently, a fast highprecision automated drilling method has become the key to the improvement of the production efficiency of suture needles with thread.

In recent years, due to its high precision, non-contact and other advantages, machine vision technology has been widely applied in various industrial detection and production fields [2-4]. Theoretically, it can meet the technical needs of

*Address correspondence to this author at the Mechanical and Electrical Engineering Department, Jiangsu Food \& Pharmaceutical Science College, Mingyuan Road, Jiangsu Huai'an 223003, China; Tel: 8613861659701 ; Email: suntiebe@163.com

1874-155X/14 automated drilling of suture needles with thread. The key of the use of machine vision technology in the production of suture needles with thread to evaluate automated end-hole drilling lies in the edge extraction of the magnified images of the needle ends to be drilled and the utilization of the highprecision positioning of edge information hole center. Traditionally, edge detection operators use differential operation between image pixels for edge extraction of images [5]; due to poor anti-noise ability, false edges or discontinuous edges easily generated [6]. Mathematical morphological edge detection uses structural elements to "detect" the signal [7]; structural elements can directly carry direction, size, chromaticity and other information and have a very good performance in identifying the known characteristic signal and filtering out noise [8]. For the highprecision positioning of hole center after edge extraction, pixel-level edge location information cannot meet the requirement of concentricity error of end holes at the micron scale $( \pm 5 \mu m \sim \pm 15 \mu m)$. In the meantime, subpixel edge positioning method based on edge detection can break through the limitations of the physical resolution. Domestic and foreign scholars have conducted a lot of research on this aspect and have presented many subpixel subdivision methods based on pixel location information. In terms of positioning precision and running time, these algorithms have both advantages and disadvantages. Among them, the algorithm put forward by Lyvers et al. in which gray spatial moments of edge are used for edge subpixel positioni

ng, can reach a theoretical pixel precision of $1 / 20$. Since it possesses advantages like minimal computational amount, good repeatability and high positioning precision, this method is widely applied [9].

Considering the technical characteristics of end-hole drilling process in the production of suture needles with 
thread, the author proposes a new edge detection method by mathematical morphology with variable structural elements, realizes subpixel edge subdivision on the basis of pixel-level edge location information, and ultimately achieves highprecision positioning of centers of holes to be drilled through least squares fitting.

\section{MATERIALS AND METHODOLOGY PRINCIPLE PRINC-ILIE FOR ACCURATE POSITIONING OF HOLE CENTER}

This system is mainly composed of quartile rotating clamping mechanism, stepping mechanism, motion control card, image acquisition card, drilling bit, CCD image sensor fixed to the drilling bit and parallel LED light source. Firstly, with the aid of CCD image sensor, the system zooms in the images of the end holes to be drilled in the imaging field of the objective lenses. Then, the images are acquired through image acquisition card and are sent to the computer for processing. The computer first turns the color image into the grayscale one, adopts the morphological method with variable structural elements to detect the edge images of the end-hole to be drilled, and uses spatial moment operators to handle the pixel-level edge points for fast subpixel subdivision. Then, least squares fitting is utilized for the extracted subpixel edge location information to determine the precise locations of the hole centers. Finally, according to the center location information, precise drilling is achieved by stepping mechanism driven by motion control card. The composition principles and work flow of the system are shown in Fig. (1).

\section{EDGE DETECTION BY MATHEMATICAL MO- RPHOLOGY WITH VARIABLE STRUCTURAL ELEMENTS}

The use of mathematical morphology with single structural elements for edge detection can both achieve the extraction of detailed information of edges and noise suppression at the same time, but some detailed characteristics of the image edges cannot be acquired because of simplicity of the structural element itself. In order to suppress the noise pollution produced in the zooming-in process of end hole images and meet the high precision positioning requirements of drilling (i.e. obtaining more detailed edge characteristics), this paper adopts variable structural elements to improve the original operators.

Assuming $f(x, y)$ as the digital images defined in the twodimensional discrete space $Z^{2}$ (denoting the set of integers), and the structural element $B(k, l) \subset Z^{2}$, the dilation and erosion operations are respectively $[10]$ :

$$
\begin{aligned}
& (f \oplus B)(x, y)=\max _{(k, l) \in B}[f(x-k, y-l)+B(k, l)] \\
& (f \bullet B)(x, y)=\max _{(k, l) \in B}[f(x+k, y+l)-B(k, l)]
\end{aligned}
$$

From the above equations, it can be known that morphological edge detection not only relates to edge detection operator, but also depends on the selection of structural element. If only one structural element is adopted, it will not be conducive to maintaining of image details and the filtering-out of different types of noises. The use of multiple structural elements can fully maintain various details of the images while filtering out different types and sizes of noises. Thus, the edge detection operator of variable structural elements is:

$$
E_{i j}(x, y)=\left\{\left[f(x, y) \oplus B_{i}(x, y)\right]-\left[f(x, y) \odot B_{j}(x, y)\right]\right\}
$$

where $B_{i}(k, l)\left(i=1,2, \ldots(N)\right.$ and $B_{j}(k, l)(j=1,2, \ldots(M)$ are selected variable structural elements.

Generally speaking, structural elements have three sizes: $3 \times 3,5 \times 5$ and $7 \times 7$. Large structural element has strong noise suppression capability, but it loses the edge details; small structural element is able to abstract more edge details, but it has weak noise suppression capability. Since the research object in this study possesses higher requirements for edge details, multiple three-point linear variable structural

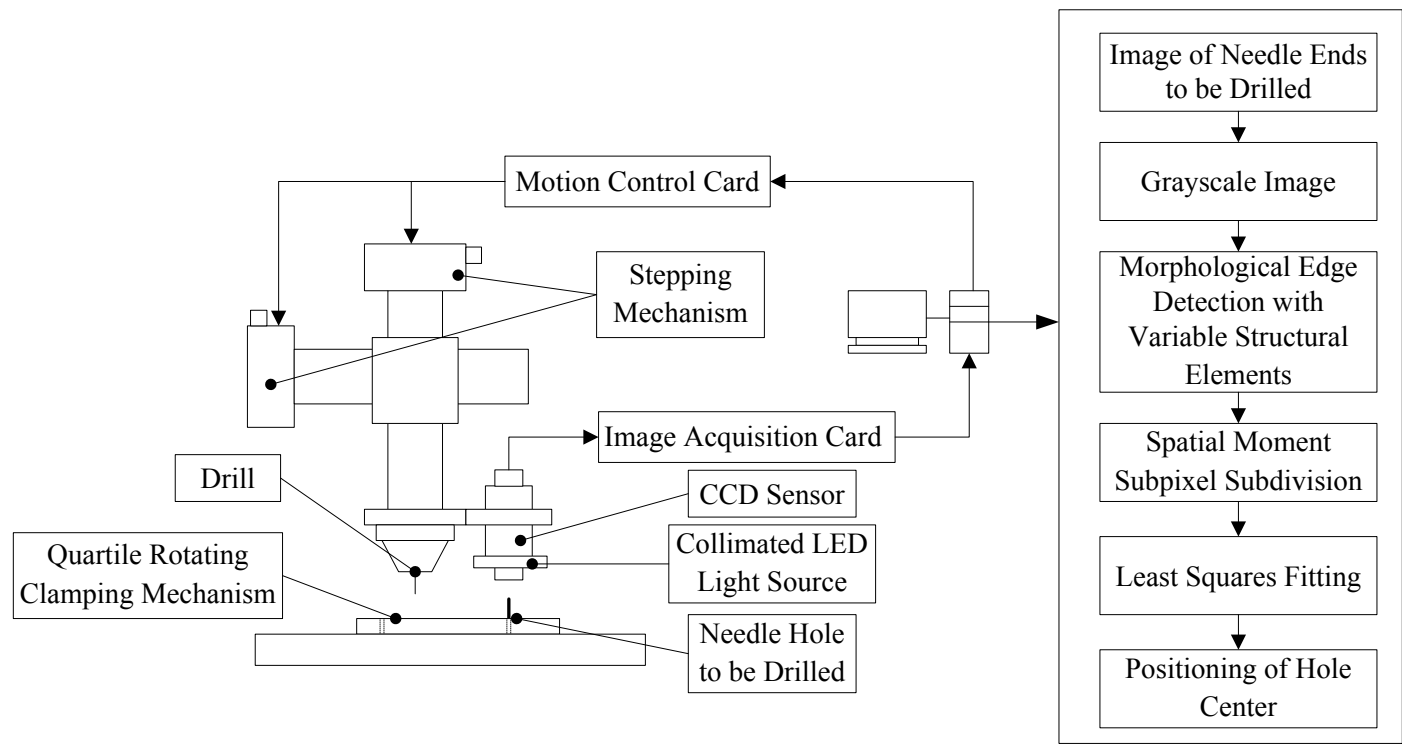

Fig. (1). Composition principles and work flow chart of the system. 
elements $(3 \times 3$ neighborhood $)$ which are symmetrical and complementary in direction and structure are selected as variable structural elements [11]. Then, the author calculates the arithmetic mean of the edge detection operator outputs of various variable structural elements to further suppress the noise and improve the edge information. The improved morphological edge detection operator $\bar{E}(x, y)$ with variable structural elements is as follows:

$\bar{E}(x, y)=\frac{1}{N}\left\{\sum_{k=1}^{m}\left[E_{k, k+m}(x, y)+E_{k+m, k}(x, y)\right]\right\}$

where $\mathrm{N}$ is the number of variable structural elements, $\mathrm{m}$ $(\mathrm{N} / 2)$ is the number of the groups of symmetrical and complementary variable structural elements, $E_{k, k+m}(x, y)$ and $E_{k+m, k}(x, y)$ are the edge images of single variable structural elements.

The structure of the three-point linear variable structural elements $(3 \times 3$ neighborhood $)$ is shown in Fig. (2).
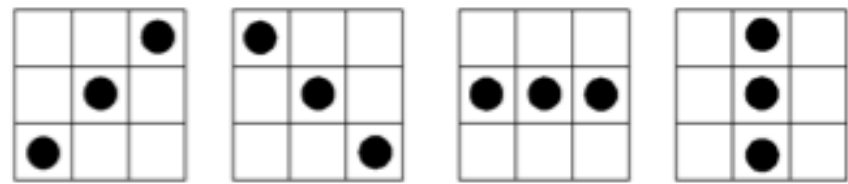

Fig. (2). Three-point linear variable structural element.

In order to verify the effectiveness of the morphological edge detection algorithm with variable structural elements in this paper, in the Matlab7.0 simulation environment, the author uses this algorithm to conduct edge detection on the images of needle end holes of 256 pixels $\times 256$ pixels which have been magnified for 50 times, and compares the detection results with those of classic Canny, Log and other edge detection operators. The edge detection results are shown in Fig. (3) and the running time of these operators is shown in Table 1.
(1)

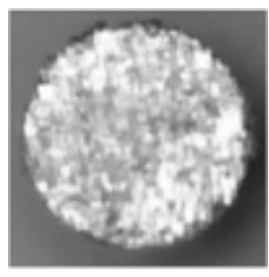

(4)

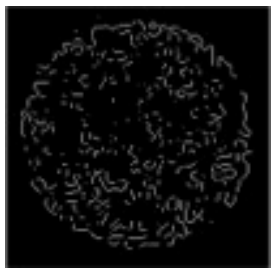

(2)

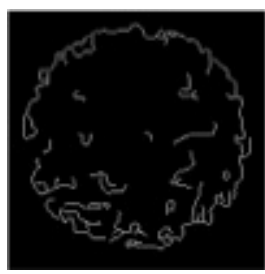

(5)

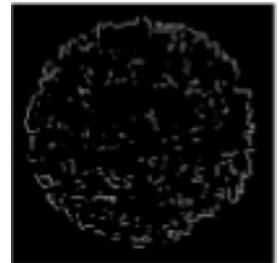

(3)

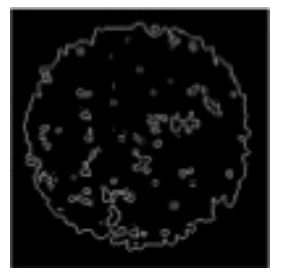

(6)

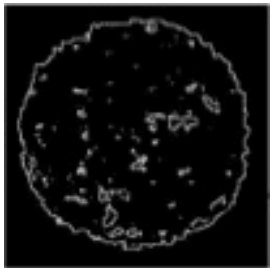

(1) Original Grayscale Image; (2) Canny Operator Edge; (3) Sobel Operator Edge; (4) Log Operator Edge; (5) Prewitt Operator Edge; (6) Algorithm in this Study.

Fig. (3). Comparison between edge detection results of different algorithms.
It can be seen from the detection results that iron fillings resulting from cutting and machining are attached to the needle end holes to be drilled, resulting in a serious noise pollution of the magnified images. Although Canny edge detection operator has strong noise suppression ability, it can only detect some effective but discontinuous edges. Directional derivative-based Log edge detection operator and Prewitt edge detection operator are severely affected by the noise and they detect a lot of false edges. Sobel edge detection operator that takes the maximum convolution can effectively suppress the noise, and can also detect too many false edges. The algorithm proposed in this study has very strong noise suppression capability and can obtain accurate, effective and continuous edges, but it has longer operation time.

\section{SPATIAL MOMENT SUBPIXEL SUBDIVISION}

In order to further improve the positioning precision, spatial grayscale moment is used for subpixel subdivision of pixel-level edge information. Theoretically, positioning precision of spatial moment method can reach 0.14 pixels [12]. Spatial moment method can establish 4 parameters for each pixel and judge the edge points based on these parameters. The 4 parameters of each pixel are the angle $\theta$ between the distance perpendicular from the center to the edge and $\mathrm{X}$-axis, the distance 1 between the center and the edge, grayscale background $\mathrm{h}$ and grayscale step height $\mathrm{k}$. The geometric meanings of various parameters are shown in Fig. (4).

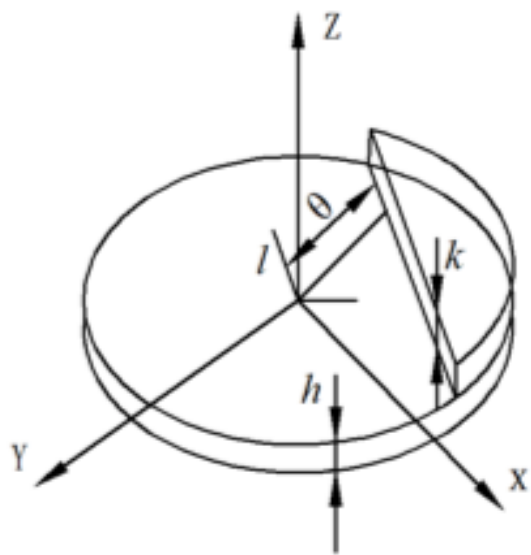

Fig. (4). Parameters in spatial moment method.

The spatial moment $f(x, y)$ of any point in the discrete image can be given by [13]:

$M_{p q}=\iint x^{p} y^{q} f(x, y) d y d x$

To reduce the dimension of spatial moment, the function $f(x, y)$ is rotated for angle $\theta$ to make it symmetrical along $X$-axis. The rotated function $f^{\prime}(x, y)$ is then obtained and the spatial moments of the images from zero-order to secondorder are figured out. In accordance with the principle that the same grayscale value remains unchanged in the image, the correspondence between spatial moments of the rotated function $f^{\prime}(x, y)$ and the original function $f^{\prime}(x, y)$ is obtained [14]: 
Table 1. Comparison of running time of the five methods.

\begin{tabular}{|c|c|c|c|c|c|}
\hline Detection Method & Canny Operator & Sobel Operator & Log Operator & Prewitt Operator & Method in this Study \\
\hline \hline Running Time (s) & 0.076 & 0.041 & 0.189 & 0.123 & 0.221 \\
\hline
\end{tabular}

$$
\left\{\begin{array}{l}
M_{00}^{\prime}=M_{00} \\
M_{10}^{\prime}=M_{10} \cos \theta+M \sin \theta \\
M_{20}^{\prime}=M_{20} \cos ^{2} \theta+M_{11} 2 \cos \theta \sin \theta+M_{02} \sin ^{2} \theta \\
M_{01}^{\prime}=-M_{10} \sin \theta+M_{01} \cos \theta \\
M_{02}^{\prime}=M_{20} \sin ^{2} \theta-M_{11} 2 \cos \theta \sin \theta+M_{02} \cos ^{2} \theta \\
M_{11}^{\prime}=\left(M_{02}-M_{20}\right) \sin \theta \cos \theta+M_{11}\left(\cos ^{2} \theta-\sin ^{2} \theta\right)
\end{array}\right.
$$

Since the function $f^{\prime}(x, y)$ is symmetrical along $X$-axis, we have:

$$
\begin{aligned}
& M_{00}^{\prime}=0 \\
& \operatorname{tg} \theta=M_{01} / M_{10}
\end{aligned}
$$

According to the second-order spatial moment of the function $f^{\prime}(x, y)$, we have:

$l=\frac{4 M_{20}^{\prime}-M_{00}^{\prime}}{3 M_{10}^{\prime}}$

$k=\frac{3 M_{10}^{\prime}}{2 \sqrt{\left(1-l^{2}\right)^{3}}}$

$h=\frac{2 M_{10}^{\prime}-k\left(\pi-2 \arcsin l-2 \sqrt{\left(1-l^{2}\right)}\right.}{2 \pi}$

Then the coordinates of the subdivided subpixel edge points are:

$$
\left\{\begin{array}{l}
x^{\prime}=x+N l \cos (\theta / 2) \\
y^{\prime}=y+N l \sin (\theta / 2)
\end{array}\right.
$$

where $(x, y)$ is the coordinate of the pixel-level edge point and $\left(x^{\prime}, y^{\prime}\right)$ is the coordinate of the subdivided subpixel edge point.

\section{CENTER COORDINATES BY LEAST SQUARES FITTING}

Based on the subpixel edge point location information obtained by spatial moment subdivision, information like the precise center and radius of the circle can be obtained, namely, the center of the needle end hole to be drilled. In this study, the author adopts least squares, a simple and practical method, for data fitting [15]. Assuming that the radius of the circle is $r$, and the coordinate of the center is $\left(x_{0}, y_{0}\right)$, then the equation of this circle is:

$$
\left(x-x_{0}\right)^{2}+\left(y-y_{0}\right)^{2}=r^{2}
$$

From (13), the error equation can be defined:
$E=\sum_{i}^{n}\left[\left(x_{i}-x_{0}\right)^{2}+\left(y_{i}-y_{0}\right)^{2}-r^{2}\right]^{2}$

According to the principle of the least squares, we have:

$$
\frac{\partial E}{x_{0}}=\frac{\partial E}{y_{0}}=\frac{\partial E}{r}=0
$$

Substituting (14) into (15) and letting $Z=x_{0}{ }^{2}+y_{0}{ }^{2}-r^{2}$, we have:

$$
\left\{\begin{array}{l}
2 \sum_{i}^{n}\left(x_{i}^{2}+y_{i}^{2}-2 x_{i} x_{0}-2 y_{i} y_{0}+Z\right)\left(-2 x_{i}\right)=0 \\
2 \sum_{i}^{n}\left(x_{i}^{2}+y_{i}^{2}-2 x_{i} x_{0}-2 y_{i} y_{0}+Z\right)\left(-2 y_{i}\right)=0 \\
2 \sum_{i}^{n}\left(x_{i}^{2}+y_{i}^{2}-2 x_{i} x_{0}-2 y_{i} y_{0}+Z\right)=0
\end{array}\right.
$$

From (16), the center coordinate $\left(x_{0}, y_{0}\right)$ and the circle radius $r$ can be obtained.

\section{EXPERIMENTAL RESULTS AND ANALYSES}

\subsection{Simulation Image Analysis}

In order to verify the effectiveness of the method proposed in this study, a simulation test is conducted on the ideal object. Specifically, the images of 10 groups of ideal circles with different radiuses and centers are constructed in the Matlab7.0 environment and the white noise whose signal-to-noise ratio is $25 \mathrm{~dB}$ is added to these images. Then, Sobel operator and the operator in this study both of which have a good performance in the edge detection of the magnified images of the needle end holes to be drilled are adopted to conduct edge detection on the 10 groups of ideal circular images. Least squares fitting method is used for fitting of the edge information from Sobel operator and the operator in this study to get the center coordinates of 2 groups of circles. After subpixel subdivision, fitting is conducted on the operator in this study to obtain one group of center coordinates. Then, the author compares the three groups of coordinates with the ideal circular centers to test the effectiveness of three methods. One group of noiseadding standard circle and edge image are shown in Fig. (5).
(1)

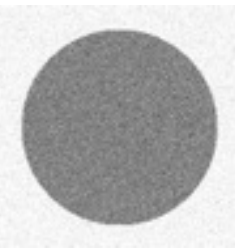

(1) Noise-adding Circle (2) Sobel Operator Edge (3) Opera-tor in this Method
Fig. (5). Noise-adding circle and edge detection result. 
Table 2. Measurement data of ideal circular image. (Unit: pixel).

\begin{tabular}{|c|c|c|c|c|c|c|c|c|}
\hline \multirow{2}{*}{ Number of Times } & \multicolumn{2}{|c|}{ Ideal Circles } & \multicolumn{2}{c|}{ Method One } & \multicolumn{2}{c|}{ Method Two } & \multicolumn{2}{c|}{ Method Three } \\
\cline { 2 - 9 } & $\boldsymbol{x}_{\boldsymbol{0}}$ & $\boldsymbol{y}_{\boldsymbol{0}}$ & $\boldsymbol{x}_{\boldsymbol{0}}$ & $\boldsymbol{y}_{\boldsymbol{0}}$ & $\boldsymbol{x}_{\boldsymbol{0}}$ & $\boldsymbol{y}_{\boldsymbol{0}}$ & $\boldsymbol{x}_{\boldsymbol{0}}$ & $\boldsymbol{y}_{\boldsymbol{0}}$ \\
\hline \hline 1 & 56 & 64 & 55.412 & 64.131 & 56.201 & 63.968 & 56.023 & 64.015 \\
\hline 2 & 80 & 95 & 79.841 & 95.832 & 80.197 & 95.302 & 79.982 & 95.017 \\
\hline 3 & 64 & 73 & 64.212 & 72.564 & 63.855 & 72.799 & 64.003 & 73.031 \\
\hline 4 & 102 & 115 & 102.601 & 114.398 & 101.876 & 114.901 & 102.009 & 115.011 \\
\hline 5 & 110 & 123 & 110.412 & 121.623 & 110.223 & 123.315 & 110.033 & 123.028 \\
\hline 6 & 71 & 69 & 70.703 & 69.854 & 70.861 & 68.913 & 71.026 & 69.019 \\
\hline 7 & 120 & 105 & 120.771 & 105.389 & 120.301 & 105.251 & 119.948 & 104.952 \\
\hline 8 & 109 & 122 & 108.699 & 123.011 & 109.199 & 122.203 & 109.021 & 122.017 \\
\hline 9 & 129 & 127 & 128.565 & 127.417 & 128.793 & 126.362 & 129.047 & 126.897 \\
\hline 10 & 130 & 101 & 129.641 & 100.552 & 130.101 & 101.203 & 130.014 & 101.023 \\
\hline
\end{tabular}

The statistical data of ideal circular center coordinates and the three groups of center coordinates detected are shown in Table 2 (Method one: Sobel operator edge; Method two: Mathematical morphological edge detection with variable structural elements; Method three: Mathematical morphological edge detection with variable structural elements and spatial moment subpixel subdivision).

The author handles the data in Table 2 and obtains the average error, maximum error and standard deviation of the detection results of the three methods, which are shown in Table 3. The error curve of the center coordinate $\left(x_{0}, y_{0}\right)$ is shown in Fig. (6).

Table 3. Error of processing results. (Unit: pixel).

\begin{tabular}{|c|c|c|c|c|c|c|}
\hline \multirow{2}{*}{} & \multicolumn{2}{|c|}{ Method One } & \multicolumn{2}{c|}{ Method Two } & \multicolumn{2}{c|}{ Method Three } \\
\cline { 2 - 7 } & $\boldsymbol{x}_{\boldsymbol{0}}$ & $\boldsymbol{y}_{\boldsymbol{0}}$ & $\boldsymbol{x}_{\boldsymbol{0}}$ & $\boldsymbol{y}_{\boldsymbol{0}}$ & $\boldsymbol{x}_{\boldsymbol{0}}$ & $\boldsymbol{y}_{\boldsymbol{0}}$ \\
\hline \hline $\begin{array}{c}\text { Average } \\
\text { Error }\end{array}$ & -0.011 & 0.081 & 0.06 & 0.022 & 0.011 & 0.001 \\
\hline $\begin{array}{c}\text { Maximum } \\
\text { Error }\end{array}$ & 0.771 & -1.337 & 0.301 & 0.315 & -0.052 & -0.048 \\
\hline $\begin{array}{c}\text { Standard } \\
\text { deviation }\end{array}$ & 0.448 & 0.725 & 0.182 & 0.282 & 0.027 & 0.041 \\
\hline
\end{tabular}
4.

The running time of the three methods is shown in Table

It can be seen from the statistical detection results of ideal noise-adding circles that for the center coordinate $\left(x_{0}, y_{0}\right)$ of the method in this study, the average errors are 0.011 pixels and 0.001 pixels, respectively; the standard deviations are 0.027 pixels and 0.041 pixels, respectively. The various errors of the center coordinates obtained by the detection and subdivision method adopted in this study are one order of magnitude lower than those of the other methods; the former has a significantly higher precision than the latter. However, since the computational amount of this method is large, its running time is longer than that of the other two methods.
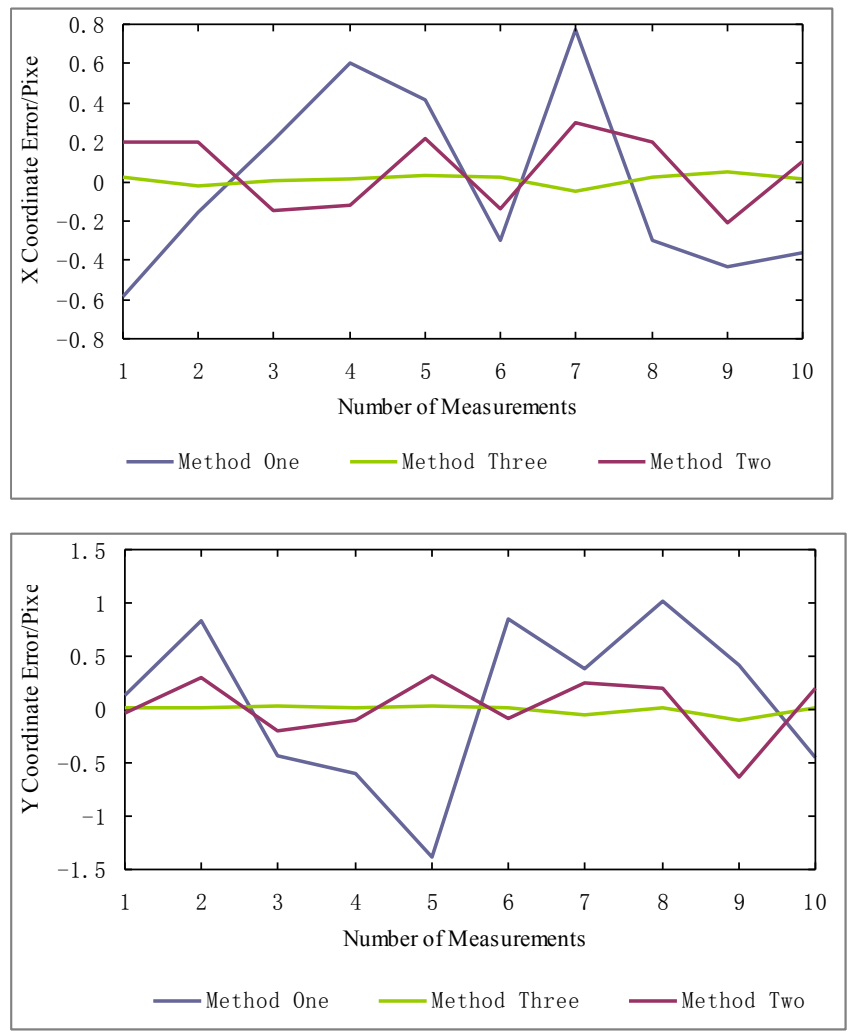

Fig. (6). Error curve of center coordinates.

Table 4. Performance comparison of three methods.

\begin{tabular}{|c|c|c|c|}
\hline Detection Method & Method One & Method Two & Method Three \\
\hline \hline Running Time $(S)$ & 0.087 & 0.032 & 0.338 \\
\hline
\end{tabular}

\subsection{Analysis of Actual Measurement Data}

The effectiveness of this algorithm should be further verified in the actual production and application. For the randomly selected 300 needles to be drilled from each of the two groups, manual drilling and drilling method proposed in this study are respectively conducted; Three indicators of hole concentricity, false detection rate (hole center 
Table 5. Statistical table of performance indicators.

\begin{tabular}{|c|c|c|c|c|}
\hline & Standard Deviation of Concentricity Error & Average Drilling Time & False Detection Rate & Miss Rate \\
\hline \hline Traditional Manual Method & $\pm 2.4 \mu \mathrm{m}$ & $0.5 S$ & 0 & 0 \\
\hline Method in this Study & $\pm 0.2 \mu \mathrm{m}$ & $0.65 S$ & $2.2 \%$ & $1.3 \%$ \\
\hline
\end{tabular}

positioning error) and miss rate (undetected needle holes to be drilled) are used to evaluate the performance of the system. The statistical data of various performance indicators in the actual measurement are shown in Table 5. The images used in the experiment are extracted by Tuo'er Corporation's Mintron MTC-63V5HP Area Array CCD Camera whose pixels are $800 \times 600$ pixels, together with the magnifying objective lens of 50 times; the pixel size is $4.65 \mu \mathrm{m} \times 4.65 \mu \mathrm{m}$ and the light source is collimated LED light source.

It can be seen from Table $\mathbf{5}$ that there is a certain gap between the measured performance and the ideal performance which mainly results from the noise pollution in the production site and the smoothing of computer images. Although this method shows a poorer performance in false detection rate and miss rate than the traditional manual method, it behaves better in drilling precision (concentricity error) and drilling time and can greatly reduce labor costs.

\section{CONCLUSION}

In accordance with the process characteristics of the endhole drilling of suture needles with thread, the author adopts non-contact machine vision technology and mathematical morphological edge detection method with variable structural elements to effectively extract the edges of the magnified images of the needle ends to be drilled. Furthermore, through subpixel subdivision, the hole center can achieve high-precision positioning. The test results of the 10 groups of ideal noise-adding circular images show that the detection method proposed in this study has a circular center positioning error of less than 0.05 pixels. The actual production experiment shows that the drilling method adopted in this study has a concentricity error $( \pm 0.2 \mu \mathrm{m})$, one order of magnitude lower than the traditional manual drilling method $( \pm 2.4 \mu \mathrm{m})$; the average drilling time can be saved up to $40 \%$. Thus, the technical needs of the end-hole drilling process in the production of suture needles with thread can be effectively met.

\section{CONFLICT OF INTEREST}

The authors confirm that this article content has no conflict of interest.

\section{ACKNOWLEDGEMENTS}

Declared none.

\section{REFERENCES}

[1] L. Hong, "Application of vibration drilling on the hole with line processing of suture needle", Modern Manufacturing Engineering, vol. 9, pp. 49-51, 2011.

[2] X. J. Wang, J. Gao and L. Wang, "A survey of subpixel object localization for image measurement", IEEE Proceedings of 2004 International Conference on Information Acquisition, Hefei, China 2004, pp. 398-401

[3] Z. Zhu, H. Yi and L. Xiaoyu, "Automatic detecting and grading method of potatoes based on machine vision", Transactions of the Chinese Society of Agricultural Engineering, vol. 28, pp. 178-183, 2012.

[4] G. Weiwei, S. Jianxin and W. Yuliang, "Algorithm of locally adaptive region growing based on multitemplate matching applied to automated detection of hemorrhages", Spectroscopy and Spectral Analysis, vol. 33, pp. 448-453, 2013.

[5] Y Kita, "Special section on machine vision and its applications: Foreword", IEICE Transactions on Information and Systems, vol. 7, pp. 1721-1725, 2012.

[6] T. Yuanyuan, L. Siyang and T. Qingchang, "Application of detecting part's size online based on machine vision", International Conference on Future Energy, Environment, and Materials, Sanya, China 2012, pp. 1948-1956.

[7] F. Lei and C. Guangcheng, "Application of mathematical morphology in the recognition and localization of circle object", Journal of Yunnan Normal University, vol. 26, pp. 8-11, 2010.

[8] W. Huifeng, Z. Guili and L. Xiaoming, "Research and application of edge detection operator based on mathematical morphology", Computer Engineering and Applications, vol. 31, pp. 223-226, 2009.

[9] L. Qiang, "Center-determination oflaser spot based on sub-pixel Positioning teehnology", Chinese Journal of Quantum Electronics, vol. 29, pp. 58-62, 2012.

[10] A. Lei, T. Jiubin and C. Jiwen, "Fast and precise center location for circle target of CCD laser autocollimator", Acta Optica Sinica, vol. 27, pp. 253-257, 2011.

[11] S. Y. B. Ke, "An edge detection algorithm of infrared image based on improved morphology", Computer and Digital Engineering, vol. 38, pp. 125-129, 2010.

[12] S. Xiaojun, Z. Yuqin and Z. Xiaohui, "Measurement of lapped surface roughness based on machine vision technique", Machine Design \& Research, vol. 26, pp. 101-103, 2010.

[13] Z. Yi, "Subpixel location of the center of laser spot based on spatial moments", Laser Infrared, vol. 35, pp. 521-524, 2012.

[14] C. An, L. Yefeng and L. Xiguo, "Inspection of hole on sheet metal parts of autobody based on spatial moment", Tool Engineering, vol.41, pp. 91-95, 2012.

[15] S. Zun-bing, C. Xian-yu and G. Guo-an, "A universal algorithm for subpixel edge detection", Journal of Shanghai Jiaotong University, vol. 41, pp. 911-915, 2011. 